\title{
A new computational strategy for analysing plastic deformation processes
}

\author{
N CHANDRASEKARAN* \\ Defence Metallurgical Research Laboratory, Kanchanbagh, Hyderabad 500258 , India \\ * Present address: Department of Mechanical Engineeringl Karnataka Regional Engineering \\ College, Surathkal 574 157, India \\ MS received 30 October 1991
}

\begin{abstract}
The paper describes a computer-based methodology for evaluating various strain and stress components within the body of a material undergoing plastic deformation. The strategy uses as input the grid distortion data, which provides the magnitudes of macroscopic particle velocities at discrete (nodal) points. To facilitate computation of the various strain-rate and strain components by explicit differentiation, B-spline functional relationships have been established for this data employing interpolating techniques. Numerical solutions obtained using this methodology have been compared with FEM predictions, which indicate that the results are in good agreement. Further, using plasticity theory, a set of partial differential equations have been established to describe the stress gradients induced due to materials processing. The mathematical formulations in the work are described only in polar coordinates, but the program VISIO can also handle the nodal displacements prescribed in rectangular coordinates too.
\end{abstract}

Keywords. Plastic deformation; computational strategy; plasticity theory.

\section{Introduction}

The establishment of metal flow lines everywhere in a deforming body is one of the first concerns of an analyst. The finite element methodology (FEM) can provide a theoretical estimate of metal flow velocities at several selected locations (nodal points). Various other theoretical methods, like slip line field (SLF), a hybrid of SLF and FEM-based techniques, etc. could also achieve the same purpose. Construction of the trajectories of metal flow by experimental techniques invariably involves monitoring distortions of grid lines marked either on the sheet metal surface or across cut-away sections of solid billets with the progress of plastic deformation. A wealth of information could also be generated by scribing lines on the free surface of deforming billets.

Sowerby and associates $(1982,1987)$ illustrate a procedure for performing a finite strain analysis in sheet metal forming operation, by comparing the shape of the original grid with that obtained after deformation. The computed points are often plotted on a forming limit diagram (FLD) described in the major vs minor strain space, in order to ascertain the severity of forming. Grid distortion data are also used to tackle several other production problems and some of those specific forming problems have been identified in the studies of Chandrasekaran and associates (Chandrasekaran 1990, 1991; Karima et al 1989, 1990). For instance, it will be possible to say whether during production, any parameters relating to press-setting, lubrication, location of blanks, etc. have been altered. Queries relating to the effect of changes made to the tooling can also be answered (Karima et al 1989, 1990).

FLD-based predictive techniques become inapplicable when applied to massive 
forming operations such as forging, extrusion, etc. Obtaining complete stress solutions become imperative, in order to use the grid distortion data to estimate damage during upset forging and to predict failure (Sowerby and Chandrasekaran 1986a, b). Other applications that demand complete solutions include predictions relating to chip segmentation during machining (Sowerby and Chandrasekaran 1989), machinability (Sathyanarayanan et al 1989; Subramanian et al 1987), etc. based on a void growth model (Sowerby and Chandrasekaran 1986a). In their study, Sowerby and Chandrasekaran (1989) had used spheroids as markers to determine strains at very high strain rates that prevail during machining.

In view of the importance narrated above, an attempt was made to develop a numerical procedure for analysing the data. The methodology uses plasticity theory dictated equilibrium equations, yield functions, associated flow rule, etc. to fulfil its objective. Thus the paper begins with a brief description of fundamentals relating to plasticity theory.

\section{Mathematical formulation based on plasticity theory}

Let $r, \theta$ and $z$ represent the radial, circumferential and axial directions, respectively of a body subjected to plastic deformation (see insert to figure 1). To facilitate analysis, let us split the continuum into smaller elements. The discretization can be achieved by taking, for instance, any longitudinal cross-section and scribing grid of lines in the plane $r-z$ (see figure 2) for the resulting finite element grid configuration. Also ensure initially that the lines are parallel to $r$ and $z$ directions. The elements then are formed by imaginary lines joining the nodal points of various cross-sections, which are concentric to the circumferential direction. A typical element is shown in figure 1.

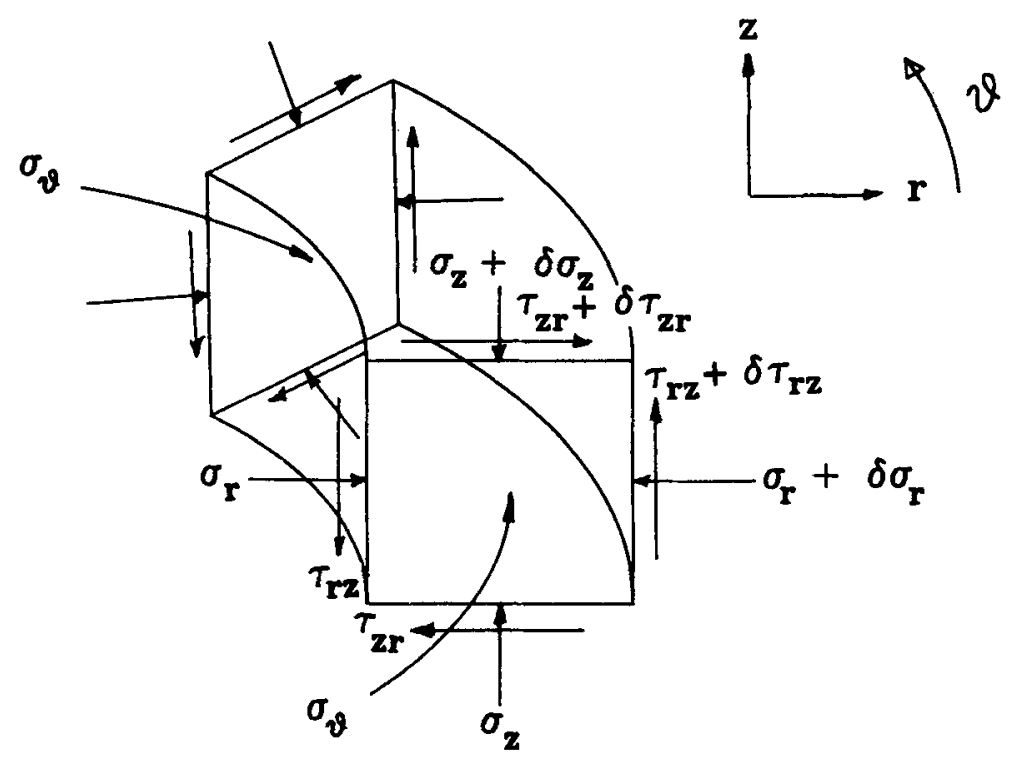

Figure 1. A 3D view of a typical axisymmetric element formed by the rectangular grid of lines; see figure 2 for a cross-sectional view. Also depicted are the various stress components acting on the front and back cross-sections. The stresses should not be misconstrued as acting only on the edges. They act on the entire internal and external curved surfaces as also on the top and bottom flat surfaces. 


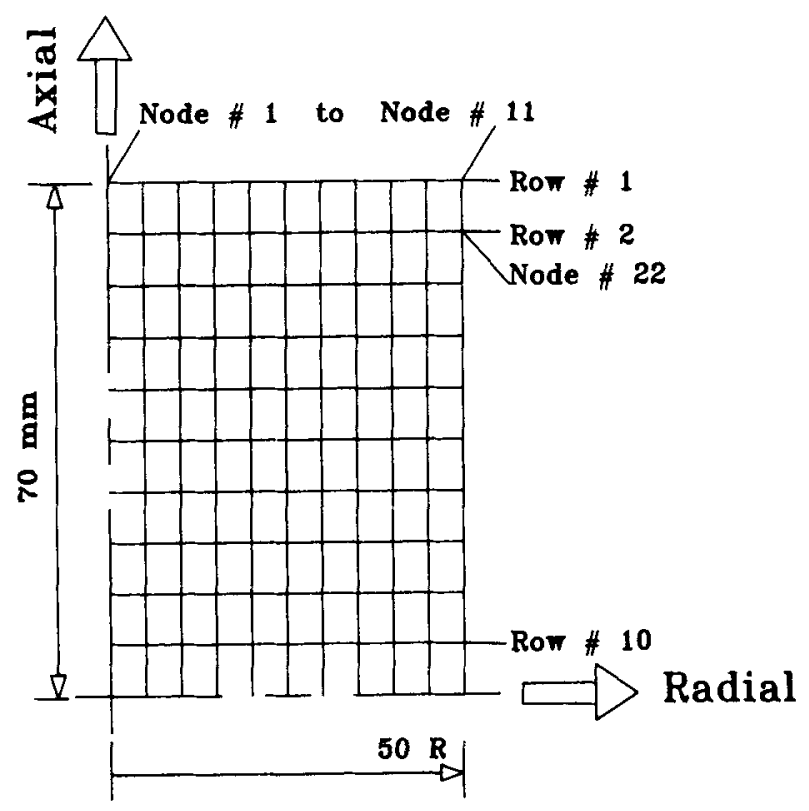

Figure 2. Finite element configuration to illustrate the row and node numbering conventions.

During deformation, let us assume that the various stress components acting on an element in the plane $r-z$ are as shown in figure 1. In the figure, $\sigma_{r}, \sigma_{\theta}$ and $\sigma_{z}$ represent the stress components acting in the $r, \theta$ and $z$ directions, while $\tau_{r z}$ denotes the shear stress acting in the plane $r z$ (normal to $r$ and along $z$ direction). Since the element cannot rotate during deformation, let us stipulate that $\tau_{r z}=\tau_{z r}$. The partial derivatives with respect to $r$ and $z$ indicate the stress gradients at point $(r, z)$.

To maintain equilibrium, resolve the forces along the $r$ and $z$ directions. The following equations in terms of the stress components and their gradients, obtal $i$

$$
\begin{aligned}
& \frac{\delta \sigma_{r}}{\delta r}+\frac{\delta \tau_{r z}}{\delta z}+\frac{\left(\sigma_{r}-\sigma_{\theta}\right)}{r}=0, \\
& \frac{\delta \tau_{r z}}{\delta r}+\frac{\delta \sigma_{z}}{\delta z}+\frac{\tau_{r z}}{r}=0 .
\end{aligned}
$$

During the course of experiments, the nodal velocity components $v_{r}$ and $v_{z}$ acting along the radial and axial directions can be calculated by comparing the initial grid with the deformed grid. Even though it may be very difficult, it is advisable to make nodal displacement measurements at closer intervals of time, due to the incremental nature of the plastic deformation process. Recall that the main objective of the work is to establish a procedure for using the velocity or displacement data and equations (1) and (2) to perform the stress analysis.

Knowing $v_{r}$ and $v_{z}$ at different coordinate points $(r, z)$ the radial, circumferential, axial and shear strain-rate components $\left(\dot{\varepsilon}_{r}, \dot{\varepsilon}_{\theta}, \dot{\varepsilon}_{z}\right.$ and $\left.\dot{\gamma}_{r z}\right)$ can be computed (Johnson and Mellor 1962), using equations (3) to (6)

$$
\begin{aligned}
& \dot{\varepsilon}_{r}=\delta v_{r} / \delta r, \\
& \dot{\varepsilon}_{\theta}=v_{r} / r,
\end{aligned}
$$




$$
\begin{aligned}
& \dot{\varepsilon}_{z}=\delta v_{z} / \delta z \\
& \dot{\gamma}_{r z}=\frac{\delta v_{r}}{\delta z}+\frac{\delta v_{z}}{\delta r}
\end{aligned}
$$

Since there is no change in volume of the material during deformation, equation (7) should hold

$$
\dot{\varepsilon}_{r}+\dot{\varepsilon}_{\theta}+\dot{\varepsilon}_{z}=0 \text {. }
$$

From associated flow rule:

$$
\frac{\dot{\varepsilon}_{r}}{\left(\sigma_{r}-\sigma_{m}\right)}=\frac{\dot{\varepsilon}_{\theta}}{\left(\sigma_{\theta}-\sigma_{m}\right)}=\frac{\dot{\varepsilon}_{z}}{\left(\sigma_{z}-\sigma_{m}\right)}=\frac{\dot{\gamma}_{r z}}{2 \tau_{r z}}=\frac{3}{2} \frac{\dot{\bar{\varepsilon}}}{\bar{\sigma}} .
$$

In (8), the axial, circumferential and radial stress components have been denoted as $\sigma_{z}, \sigma_{\theta}$ and $\sigma_{r}$. Then, the mean stress $\sigma_{m}$ by definition is:

$$
\sigma_{m}=\frac{1}{3}\left(\sigma_{r}+\sigma_{\theta}+\sigma_{z}\right)
$$

Also in (8), the effective (von-Mises) stress $\bar{\sigma}$ and strain-rate $\dot{\bar{\varepsilon}}$, in terms of stress and strain-rate components are given by

$$
\begin{aligned}
& \bar{\sigma}=\frac{1}{\sqrt{2}}\left[\left(\sigma_{r}-\sigma_{\theta}\right)^{2}+\left(\sigma_{\theta}-\sigma_{z}\right)^{2}+\left(\sigma_{z}-\sigma_{r}\right)^{2}+3 \tau_{r z}^{2}\right]^{1 / 2} \\
& \dot{\bar{\varepsilon}}=\left[\frac{2}{3}\left(\dot{\varepsilon}_{r}^{2}+\dot{\varepsilon}_{\theta}^{2}+\dot{\varepsilon}_{z}^{2}+\frac{1}{2} \dot{\gamma}_{r z}^{2}\right)\right]^{1 / 2}
\end{aligned}
$$

The plastic deformation behaviour of the material can be characterized by the equation (any other form would not matter)

Here,

$$
\bar{\sigma}=K \bar{\varepsilon}^{n} \text {. }
$$

$$
\bar{\varepsilon}=\int \dot{\bar{\varepsilon}} \mathrm{d} t
$$

In (12), $K$ is the material strength parameter and $n$ the work-hardening index. The following four equations can be obtained from (8):

$$
\begin{aligned}
& \dot{\varepsilon}_{\mathrm{r}}=\frac{3}{2} \frac{\dot{\bar{\varepsilon}}}{\bar{\sigma}}\left(\sigma_{r}-\sigma_{m}\right), \\
& \dot{\varepsilon}_{z}=\frac{3}{2} \frac{\dot{\bar{\varepsilon}}}{\bar{\sigma}}\left(\sigma_{z}-\sigma_{m}\right), \\
& \dot{\varepsilon}_{\theta}=\frac{3}{2} \frac{\dot{\bar{\varepsilon}}}{\bar{\sigma}}\left(\sigma_{\theta}-\sigma_{m}\right), \\
& \dot{\gamma}_{r z}=3 \frac{\dot{\bar{\varepsilon}}}{\bar{\sigma}} \tau_{r z} .
\end{aligned}
$$

From (14) and (15)

$$
\begin{aligned}
\left(\dot{\varepsilon}_{z}-\dot{\varepsilon}_{r}\right) & =\frac{3}{2} \cdot \frac{\dot{\bar{\varepsilon}}}{\bar{\sigma}}\left(\sigma_{z}-\sigma_{r}\right), \\
\sigma_{z} & =\sigma_{r}+\frac{2}{3} \frac{\bar{\sigma}}{\overline{\bar{\varepsilon}}}\left(\dot{\varepsilon}_{z}-\dot{\varepsilon}_{r}\right) .
\end{aligned}
$$


Differentiating the above equation and (17), we get

and

$$
\frac{\delta \sigma_{z}}{\delta r}=\frac{\delta \sigma_{r}}{\delta r}+\frac{2}{3} \frac{\delta}{\delta r}\left[\frac{\bar{\sigma}}{\overline{\bar{\varepsilon}}}\left(\dot{\varepsilon}_{z}-\dot{\varepsilon}_{r}\right)\right]
$$

$$
\frac{\delta \tau_{r z}}{\delta z}=\frac{1}{3} \frac{\delta}{\delta z}\left[\frac{\bar{\sigma}}{\overline{\dot{\varepsilon}}} \dot{\gamma}_{r z}\right] .
$$

Substituting these values in (1) and (2), we get,

$$
\begin{aligned}
& \frac{\delta \sigma_{z}}{\delta r}=\frac{2}{3} \frac{\delta}{\delta r}\left[\frac{\bar{\sigma}}{\overline{\bar{\varepsilon}}}\left(\dot{\varepsilon}_{z}-\dot{\varepsilon}_{r}\right)\right]-\frac{1}{3} \frac{\delta}{\delta r}\left(\frac{\bar{\sigma}}{\overline{\bar{\varepsilon}}} \dot{\gamma}_{r z}\right)-\frac{2}{3} \frac{\bar{\sigma}}{\overline{\bar{\varepsilon}}}\left(\frac{\dot{\varepsilon}_{r}-\dot{\varepsilon}_{\theta}}{r}\right) \\
& \frac{\delta \sigma_{z}}{\delta z}=-\frac{1}{3} \frac{\delta}{\delta z}\left(\frac{\bar{\sigma}}{\overline{\bar{\varepsilon}}} \dot{\gamma}_{r z}\right)-\frac{1}{3} \frac{\bar{\sigma}}{\overline{\bar{\varepsilon}}} \dot{\gamma}_{r z} .
\end{aligned}
$$

To evaluate the axial stress component, the above set of equations have to be integrated numerically. Also from (1) we get

$$
\frac{\delta \sigma_{r}}{\delta r}=-\frac{1}{3} \frac{\delta}{\delta z}\left(\frac{\bar{\sigma}}{\overline{\bar{\varepsilon}}} \dot{\gamma}_{r z}\right)-\frac{2}{3} \frac{\bar{\sigma}}{\overline{\bar{\varepsilon}}}\left(\frac{\dot{\varepsilon}_{r}-\dot{\varepsilon}_{\theta}}{r}\right) .
$$

The radial stress distribution can be obtained by integrating (20). Similarly, appropriate substitutions in (1) and (2) can be made to determine the circumferential and shear stress components (refer to (21) and (22)).

$$
\left(\sigma_{r}-\sigma_{\theta}\right)=\frac{2}{3} \frac{\bar{\sigma}}{\dot{\bar{\varepsilon}}}\left(\dot{\varepsilon}_{r}-\dot{\varepsilon}_{\theta}\right)
$$

or

$$
\begin{aligned}
& \sigma_{\theta}=\sigma_{r}-\frac{2}{3} \frac{\bar{\sigma}}{\overline{\bar{\varepsilon}}}\left(\dot{\varepsilon}_{r}-\dot{\varepsilon}_{\theta}\right) \\
& \tau_{z z}=\frac{1}{3} \frac{\bar{\sigma}}{\dot{\bar{\varepsilon}}} \dot{\tau}_{z z}
\end{aligned}
$$

A computer program VISIO was written to perform a complete stress analysis using the above equations.

\section{Computational strategy}

Recourse to a rigid plastic finite element code developed by Kobayashi et al (1989) was made in order to perform a plastic deformation analysis of an axi-symmetric upsetting process. Due to symmetry, only one quadrant of the cylindrical billet was analysed (see figure 2). The numbering convention was such that row numbers increase when proceeding from top to bottom (refer to figure 2). Notice that the row in contact with the top platen is called Row \#1. The nodes are numbered 1 to 121 (11 rows with 11 nodes/rows) as indicated in the figure. Observe that the number of rows and columns are 11 each.

Even though the avowed objective of the work is to seek stress solutions by solving explicit plasticity theory-based equations, the reasons for seeking FEM predictions are two-fold. First, FEM provides strain-rate and stress distributions within the deforming body, which can be used for comparative purposes to test our formulation. 
Secondly, it predicts the geometries of the grids, which distort with deformation. The geometry should be exactly the same as that one would obtain through experiments. It would not matter, even if it is not so, because the problem is to obtain solutions given the grid distortion.

\subsection{Calculation of strain-rates and strains}

The computational strategy begins with extracting from FEM outputs the radial and axial velocities of the particles (they bear no resemblance to microscopic particles) at all the nodal points. To impart clarity, the procedure is outlined taking the data pertaining to radial velocity component.

A program called SMOOT downloaded from Cyber (at McMaster University, Canada) and ported to a PC was employed to fit B-spline cubic interpolating functions to the different characteristics. SMOOT can also calculate the derivatives at any intermediate point. Using this technique B-spline interpolating functions were generated to describe the variations of velocities with radius and height. To begin with, a total of 22 functional relationships, one each for 11 rows and 11 columns, were established. Observe that this has to be done because FEM provides output at discrete points and not in the form of continuous functions. A similar procedure was adopted to handle the axial velocity data. It can be inferred from figures 3 and 4 that

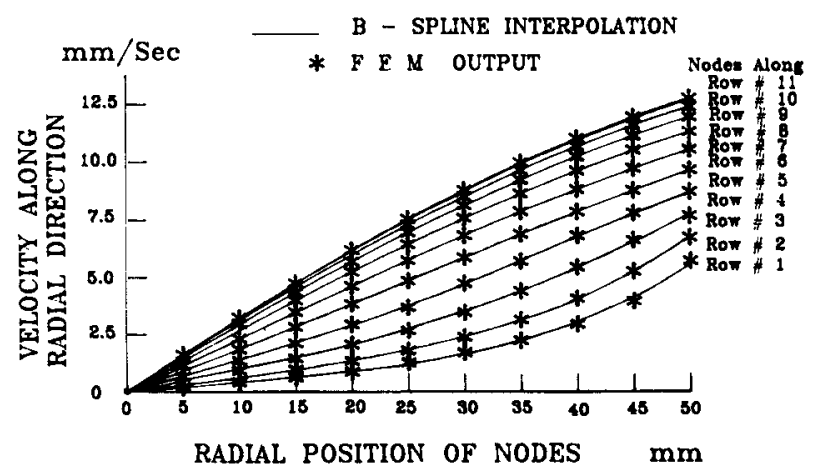

Figure 3. Characteristics describing the radial nodal velocities of different nodes. B-spline interpolated values used as input to VISIO are compared with FEM output.

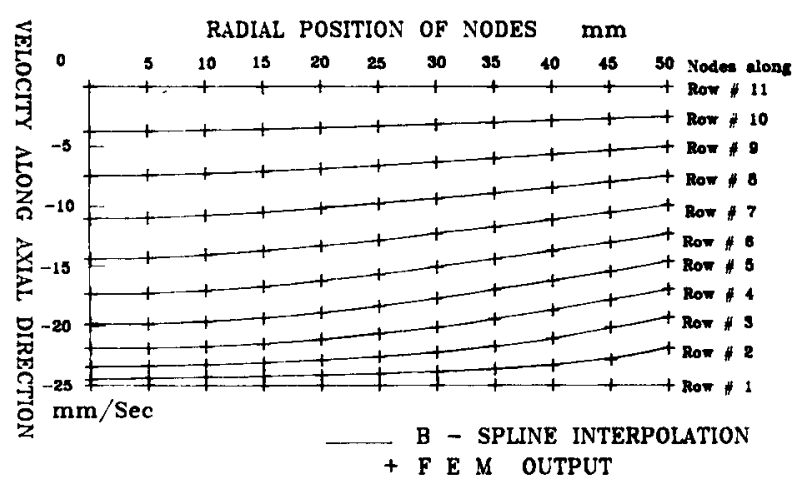

Figure 4. Characteristics describing the axial nodal velocities of different nodes. B-spline interpolated values used as input to VISIO are compared with FEM output. 
the velocities computed using the interpolating functions (shown as continuous lines) are compatible with FEM outputs at all the nodal points. In the above figures, the magnitudes of the radial and axial velocity components calculated using the FEM technique have been represented using star and plus symbols respectively. There are 11 characteristics in the figures representing rows 1 to 11 and the nodes on these rows are located $5 \mathrm{~mm}$ apart along $r$ direction.

The fact that SMOOT also computes derivatives facilitates evaluation of the radial, circumferential, axial and shear strain-rate components, by direct substitution of the computed derivatives into equations (3) to (6). Further using (11), the effective strain-rate was also calculated. The computed axial and effective strain rate variations are shown in figures 5 and 6 . The results obtained based on the current strategy are in good agreement with FEM predictions. The above figures depict strain-rates of

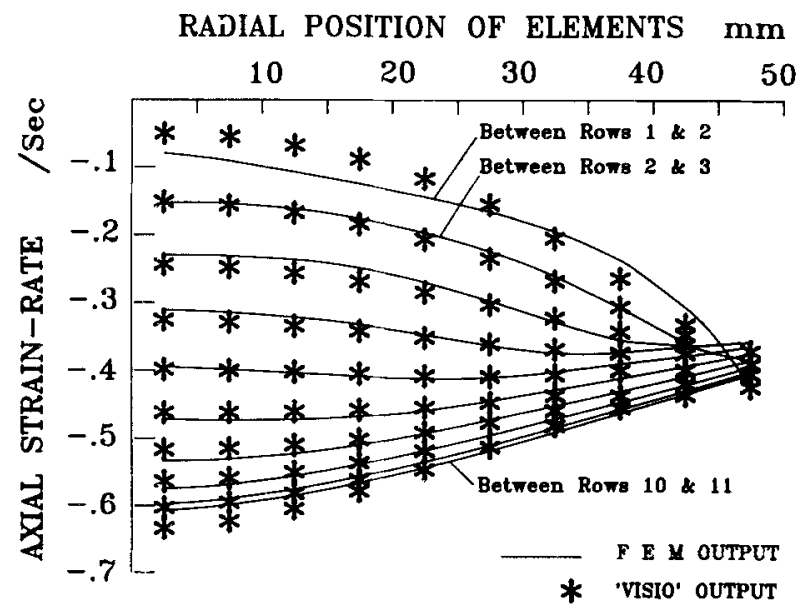

Figure 5. Axiai strain-rates computed after generating spline functions. The values are compared with FEM output.

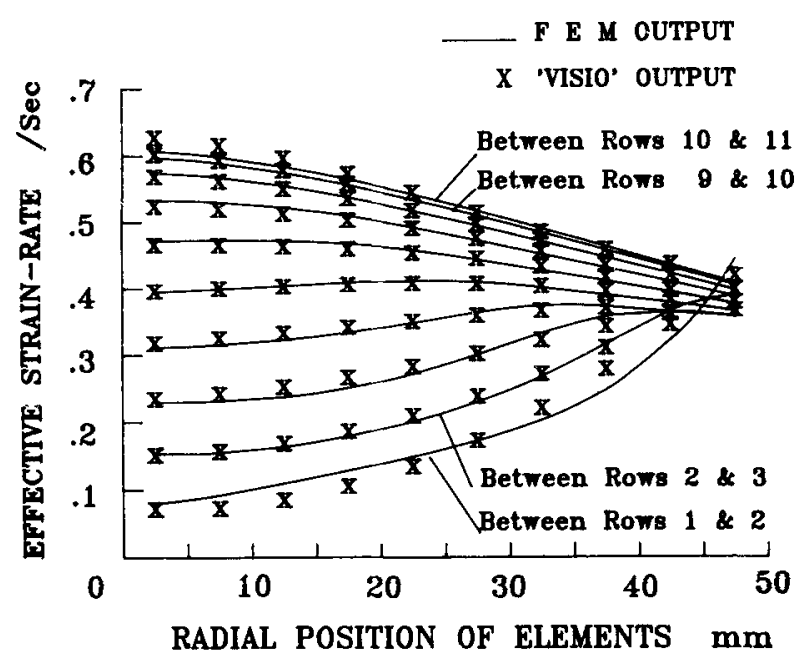

Figure 6. Representative strain-rates computed after generating spline functions. The values are compared with FEM output. 
various elements. These elements lie between adjacent rows; for example between rows 1 and 2, 2 and 3, etc. The radial distance between the vertical $z$ axis and the centre of the first column of elements is $2.5 \mathrm{~mm}$.

It is heartening to note that the axial and effective strain-rate components have similar characteristics, because a low friction factor of $m=0.25$ was assumed in the calculations. Needless to point out that in the absence of friction the two characteristics will absolutely overlap. Also, adjacent to the free surface the strain values are nearly the same. The maxima occur at the centre, while the minima at the middle of the top surface. The free surface values are approximately the average. In spite of these low magnitudes, it will be shown in subsequent calculations that higher circumferential tensile stresses develop in the free surface, making it prone to fracture.

Let us assume that $\delta t$ is the incremental time (the time taken for each deformation step) that has brought about the grid distortion. Then, the increments in various strain comp nents during this time interval can be evaluated by integrating the rate components using equations that are similar to (13). These strains are logarithmic and hence are additive. Thus the various total strain components can also be computed, from the rate components.

Next, let us see how the strain distributions can be obtained knowing the rate variations. In the current example, we have assumed that the top platen was moving at a velocity of $25 \mathrm{~mm} / \mathrm{s}$ and that $\delta t$ was $0.056 \mathrm{~s}$. At the beginning of the calculations, to compute the material flow velocities, the geometries of the grids obtained after the fourth and fifth incremental steps were compared. Assuming linear strain path, the strain component at the end of the fifth step will be $(5 * 0.056=) 0.28$ times the rate component shown in figures 5 and 6.

\subsection{Calculation of stresses}

The next step is to evaluate the shear stress and the three deviatoric stress components. To achieve this, the known equivalent strain-rate and stress values have been substituted in equation (14) through (17). Let us also pick (18) to illustrate how the numerical integration was performed. The right side of this equation has three terms. The first two terms are partial derivatives of functions of radial distance $r$. These functions were generated using B-spline interpolations approach based on SMOOT. The derivatives were also evaluated at all the nodes. The variation of axial stress with $r$ was then calculated. Again bear in mind that there are several ways of extracting the axial stress component. Take for instance node no. 13 (row \#2) in figure 2 . The starting point for the stress calculations is node no. 121 (row \#11), which is on the free surface, because the radial stress there is zero. To get to node no. 13, from 121, the axial stress gradient along $r$ for row $\# 11$ has to be known. This will enable the calculations of stresses at node no. 112, which is directly beneath node no. 13. To proceed from 112 to 13 , the axial stress gradient along $z$ for that particular column has to be known. Alternatively, we could have proceeded up along the free surface to row no. 2 and radially inwards along row \#2 to node no. 13. The important point is that there are scores of ways of doing the calculations, but the results have to be compatible. Fortunately, this has proved to be an excellent check for the computational procedure and not a hindrance.

In figure 7 are shown the computed axial stress values. Observe that the characteristics is similar to figures 5 and 6 . To digress and make an important point, 


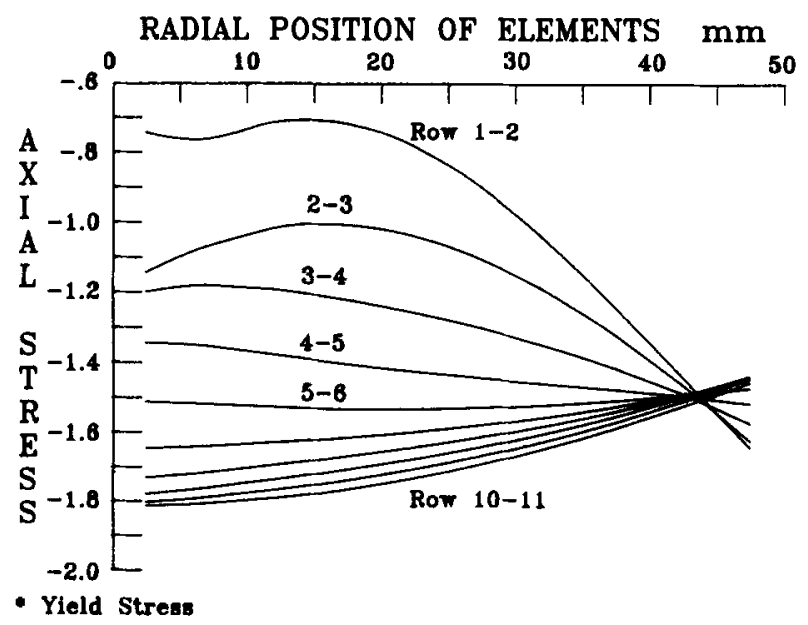

Figure 7. Axial stress distribution obtained using plasticity theory-based approach.

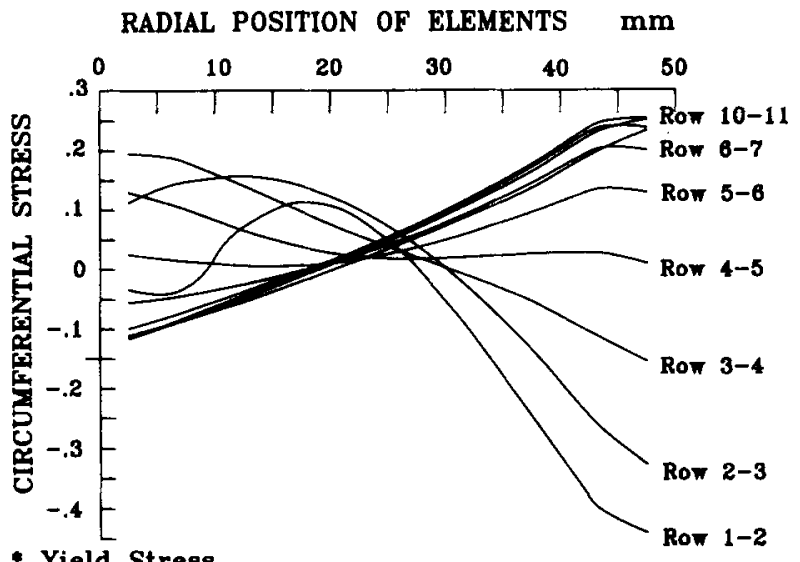

\section{- Yield Stress}

Figure 8. Circumferential stress distribution obtained using plasticity theory-based approach.

let the work-hardening index for the material be assumed to be $0 \cdot 182$. The yield stress then becomes $(0.01 * * 0 \cdot 182=) 0.4325$ times the material strength parameter $K$ given in (12). All the stresses were normalized with respect to yield stress. Instead, if one desires normalization w.r.t. $K$ value, $y$-axis has to be scaled down by 0.4325 times.

In figure 8 , the extracted circumferential stress components have been shown. Observe that in the elements near the free surface (radial position close to $50 \mathrm{~mm}$ ), which are between rows 7 to 11, the maximum build-up of circumferential tensile stresses occur. Sowerby and Chandrasekaran $(1986 \mathrm{a}, \mathrm{b})$ have explained the significance of this build-up, vis-a-vis the phenomenon of ductile fracture.

Figures 9 to 10 depict the radial and shear stress variations. Observe that the shear stresses are higher, closer to the workpiece-tool interface. This is physically realistic because, at the interface the friction impedes the radial movement of the work and causes to shear most. The representative stress characteristics is shown in figure 11 .

One of the major concerns in numerical methods often relates to the magnitude 


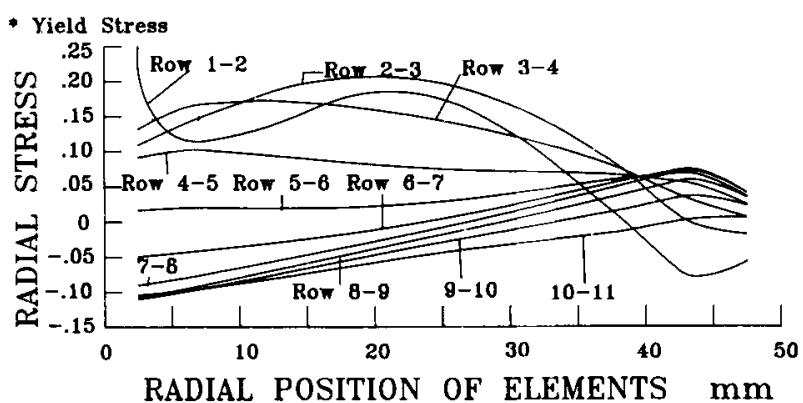

Figure 9. Radial stress distribution obtained using plasticity theory-based approach.

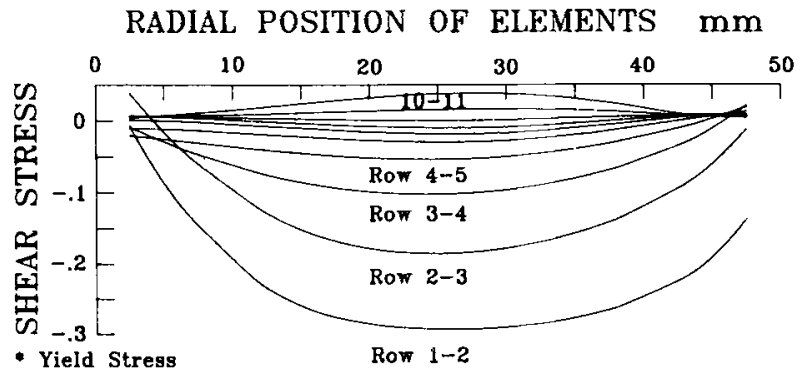

Figure 10. Shear stress distribution obtained using plasticity theory-based approach.

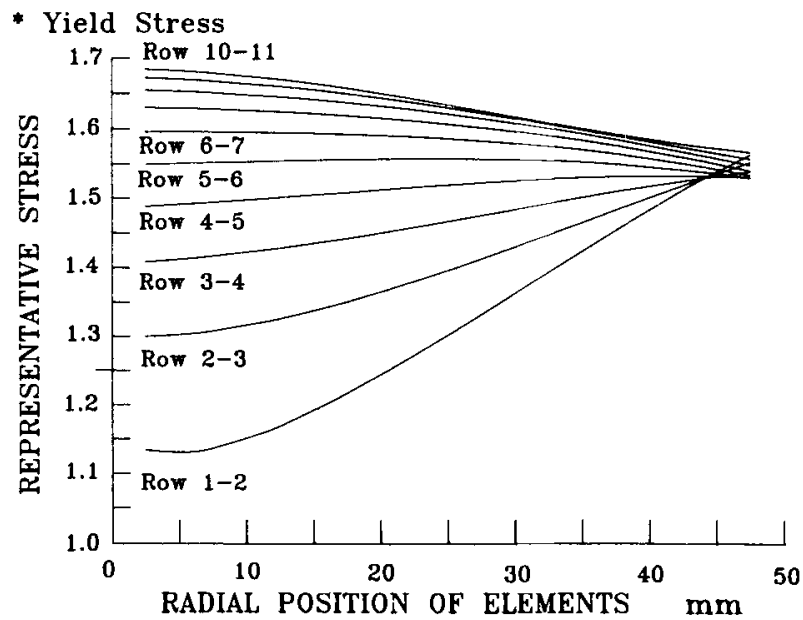

Figure 11. Representative stress distribution obtained using plasticity theory-based approach.

of error the techniques introduce during computation. Chandrasekaran et al (1982) addressed this query and have demonstrated that the influence of strain history (strain-path) on the magnitude of total strain (calculated by integration) is marginal. They further established that since the magnitude of the stress components is related only to the instantaneous slope of the strain-path the errors are not cumulative. An analyst has to make sure that smoothing techniques do not introduce abrupt changes in first derivatives of the major vs minor strain curves. B-spline interpolation 
techniques are ideal for ensuring smoothness and thus for minimizing computational errors (see Sowerby and Chandrasekaran 1986a, b, 1989; Sathyanarayanan et al 1989).

A second concern will be whether the errors are additive since the stress computations have been performed proceeding from the free surface inwards. The fact that the magnitude of stress components has been computed with minimum error should ensure that the stress gradients too are accurate. This technique is superior to finite difference-based partial differential equation solution schemes because errors can be minimized simply by ensuring smoothness of the grid distortion or the strain history characteristics.

\section{Conclusions}

The equations describing the equilibrium of an element in terms of the stress components, together with plasticity theory-based equations have been utilized to construct a mathematical formulation for performing a complete strain and stress analysis. The main features of the program VISIO have been outlined by means of a step-by-step procedure. The results obtained using this strategy have been compared with FEM predictions. The ensuing agreements indicate that plastic deformation processes can be confidently analysed employing the new technique.

The important advantage is that the strategy will be a boon to experimentalists. It can handle even very complicated shapes. The cost of acquiring or developing an exclusive FEM code will increase with the complexity of the geometry. Even the published grid distortion data obtained based on FEM techniques, can be further analysed with this simple tool.

\section{References}

Chandrasekaran N 1990 Applications of modelling techniques in metal forming, Presented at 44th Annual Technical Meeting, Indian Institute of Metals, Trichy

Chandrasekaran N 1991 Curr. Sci. 61819

Chandrasekaran N, Brownrigg A, Duncan J L, Embury J D and Sowerby R 1982 Scr. Metall. 16697

Johnson W and Mellor P B 1962 in Plasticity for mechanical engineers (New York: von Nostrand)

Karima M, Chandrasekaran N and Tse W 1989 J. Mater. Shaping Technol. 7169

Karima M, Chandrasekaran N and Tse W 1990 A novel approach for trouble-shooting stamping problems based on the concept of process signatures; Proc. SAE 1990 International Congress, Detroit, USA, pp 13-24

Kobayashi S, Soo-Ik Oh and Altan T 1989 in Metal forming and the finite element method (London: Oxford University Press)

Sathyanarayanan G, Kane G E, Sowerby R and Chandrasekaran N 1989 J. Mater. Shaping Technol. 7161

Sowerby R and Chandrasekaran N 1986a Mater. Sci. Engg. 7915

Sowerby R and Chandrasekaran N 1986b Mater. Sci. Engg. 7927

Sowerby R and Chandrasekaran N 1989 Mater. Sci. Engg. A119 219

Sowerby R, Chu E and Duncan J L $1982 \mathrm{~J}$. Strain Anal. 795

Sowerby R, Chandrasekaran N, Chen X, Rooks M J and Correa P 1987 The development for computer aids for sheet metal stampings, Proc. III International Conf. on CAD/CAM and FEM in metal forming, Stafford, UK (London: Pergamon Press) pp 187-203

Subramanian S V, Kay D A R, Chandrasekaran N and Sowerby R 1987 Design of clean steel for high strain rate machining, Proc. Int. Conf. on Strategies for automation of machining: $M$ aterials and Processes, ASM International, Orlando, Florida, USA, pp 27-42 